\title{
Electrically controlled resistive switching assisted active ultra-broadband optical tunability in the infrared
}

\author{
Enes Battal ${ }^{1,2}$, Ayşe Özcan ${ }^{2}$, and Ali Kemal Okyay ${ }^{1,2}$ \\ ${ }^{1}$ Bilkent University, Department of Electrical and Electronics Engineering, Bilkent, TR-06800, Ankara, Turkey \\ ${ }^{2}$ UNAM - Institute of Materials Science and Nanotechnology, Bilkent University, TR-06800, Ankara, Turkey \\ *Tel:+90 (312) 290 1557,e-mail: battal@ee.bilkent.edu.tr
}

\begin{abstract}
We present an electrically tunable optical device with ultra-broadband tunability operating in $2-10 \mu \mathrm{m}$ spectrum. We also, for the first time, optically observe resistive switching behavior in reflection measurements under electrical bias.
\end{abstract}

\section{INTRODUCTION}

Active control of the optical properties of materials have always been of significance due to its various applications such as tunable optical filters [1], beam steering [2], adaptive sensors [3] and holographic devices [4]. Recently, nonlinear [5], electro-optic [6], magneto-optic [7], phase change [8] materials, doped semiconductors[9], liquid crystals [10], micromechanical structures [11] have been realized in order to gain control over the refractive index of an optical media. Yet, achieving optical tunability in a broad spectrum remains a challenge.

In this study, we experimentally demonstrate an electrically tunable optical device exhibiting ultra-broadband tunability characteristic in the mid-infrared spectrum. The proposed device consists of a $\mathrm{p}-\mathrm{n}$ junction formed by $\mathrm{ZnO} / \mathrm{p}-\mathrm{Si}$ and an inherent memristor realized by addition of a metallic top contact $(\mathrm{Al} / \mathrm{ZnO} / \mathrm{p}-\mathrm{Si})$. We obtained a change of about $7 \%$ in the reflection spectra under electrical bias and also, for the first time, observed a hysteresis in the reflection spectra due to resistive switching.

\section{FABRICATION \& MEASUREMENTS}

On top of a highly p-type Silicon substrate, we deposited a blanket of $\mathrm{ZnO}$ thin film with 300 cycles of $200^{\circ} \mathrm{C}$ atomic layer deposition (ALD) using Cambridge Nanotech Savannah Thermal ALD. Then, the $\mathrm{ZnO}$ layer is isolated into an area of

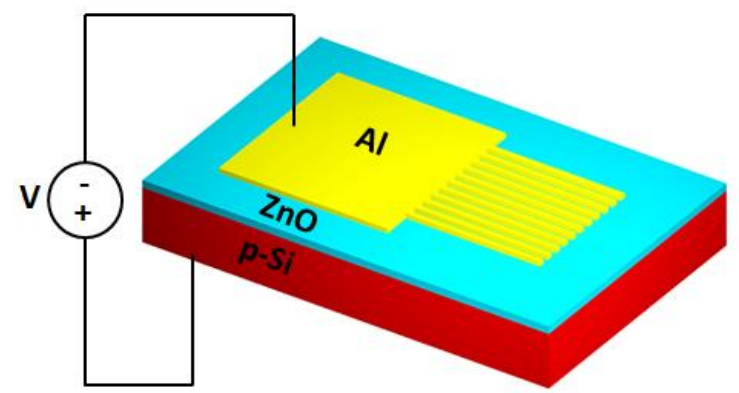

Figure 1 - Fabricated device structrure along with the voltage bias configuration
$600 \mu \mathrm{m} \times 300 \mu \mathrm{m}$ using optical lithography and wet etching with $\mathrm{H}_{2} \mathrm{SO}_{4}: \mathrm{H}_{2} \mathrm{O}(2: 100)$ solution. The deposited $\mathrm{ZnO}$ layer is previously shown to be highly doped with electron concentrations on the order of $10^{19} \mathrm{~cm}^{-3}$ [12]. An Aluminum contact pad with fingers has been formed with consecutive optical lithography, thermal evaporation and lift-off processes. The final form of the fabricated structure is shown in Fig. 1. Electrical measurements are performed using top $\mathrm{Al}$ and bottom Si layers as contacts with Keithley 2400 Source Measure Unit. Optical measurements from the area with metallic fingers are performed using a Bruker Vertex 70 Fourier Transform Infrared (FTIR) spectrometer with a Hyperion 2000 microscope.

\section{RESULTS AND DISCUSSION}

A voltage sweep loop between $-6 \mathrm{~V}$ and $6 \mathrm{~V}$ is applied to the device with $1 \mathrm{~V}$ steps. At each voltage step, reflection spectra of the device are measured in the spectral range of $2-10 \mu \mathrm{m}$, concurrently. This measurement loop is carried out twice for consistency. In Figure 2, reflection spectra at the wavelength of $2 \mathrm{um}$ as a function of bias voltage is depicted for both of the measurement loops. A maximum change of about $7 \%$ in the reflection observed. Considering the highly doped nature of both $\mathrm{Si}$ and $\mathrm{ZnO}$ layers, the optical characteristics of these layers in the spectrum of interest is dependent on the amount of free carriers according to Drude oscillator model in this spectrum. The depletion width increases under reverse bias and reduction in the number of free carriers in both $\mathrm{ZnO}$ and $\mathrm{Si}$ and induces a change in the reflection spectrum. Under forward bias, similar mechanism occurs but with an increasing concentration of free carriers in both layers with shallower

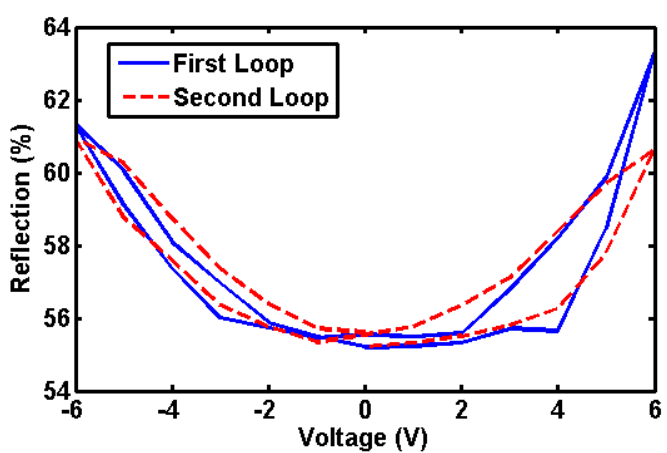

Figure 2 - A repeatable optical hysteresis in the reflection spectrum is observed when the bias voltage is swept in a loops. 


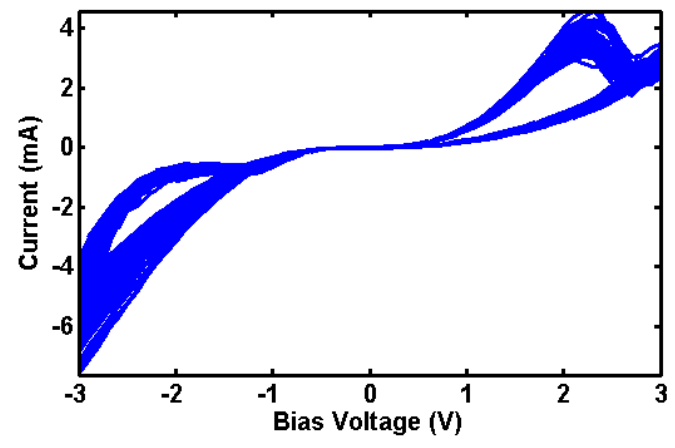

Figure 3 - A resistive switching behavior is clearly observed in the current-voltage measurements.

depletion region. Therefore, any change in the number of free carriers would contribute to the change in refractive index of the dielectric layers, and alter the reflection spectrum.

We also observed a repeating hysteresis in the reflection spectra of the device. In order to evaluate such a hysteretic behavior, we measured the current-voltage (I-V) relationship with a voltage sweep. A repeatable memristive hysteresis is observed in the I-V relationship in Fig. 3. For both optical and electrical hysteresis, a contrast ratio of about 4 is observed. Due to very high doping of $\mathrm{ZnO}$ layer, the contrast ratio is observed to be very low. Such an optical hysteresis of a memristive device is observed for the first time in the literature.

We also investigated the broadband characteristics of the electrical tunability of the reflection spectra along with its resistive switching behavior. For the wavelength range of 2$10 \mu \mathrm{m}$ and bias voltages between $-6 \mathrm{~V}$ and $6 \mathrm{~V}$ with $3 \mathrm{~V}$ steps starting from $0 \mathrm{~V}$, the reflection spectra is depicted in Figure 4. When the bias voltage is increased from $0 \mathrm{~V}$ up to $6 \mathrm{~V}$ or decreased down to $-6 \mathrm{~V}$, the entire reflection spectrum increases by about $5.5 \%$. Such tunability in a broadband spectral range is obtained with bias voltages that fall into CMOS compatible region. The optical hysteresis behavior observed in Figure 2 can also be seen in this figure such that the reflection spectrum is dependent on its past of the electrical bias. The reflection spectrum at $3 \mathrm{~V}$ (or $-3 \mathrm{~V}$ ) does not differ from that of $0 \mathrm{~V}$ unless $3 \mathrm{~V}$ (or $-3 \mathrm{~V}$ ) is applied after returning from $6 \mathrm{~V}$ (or $-6 \mathrm{~V}$ ). We further investigated the mechanisms behind the optical observation of resistive switching and constant change in the overall reflection spectra.

\section{CONCLUSION}

We demonstrated a broadband electrically tunable optical reflection device operating in the spectral range of $2-10 \mu \mathrm{m}$. We observed change in the reflection spectra as high as $6 \%$. The bias voltages that are necessary for tuning are on the order of several volts. We also optically observed the memristive hysteresis of metal-oxide based structure. This study paves the way for the utilization of resistive switching behavior along with broadband tunability in optoelectronic device applications.

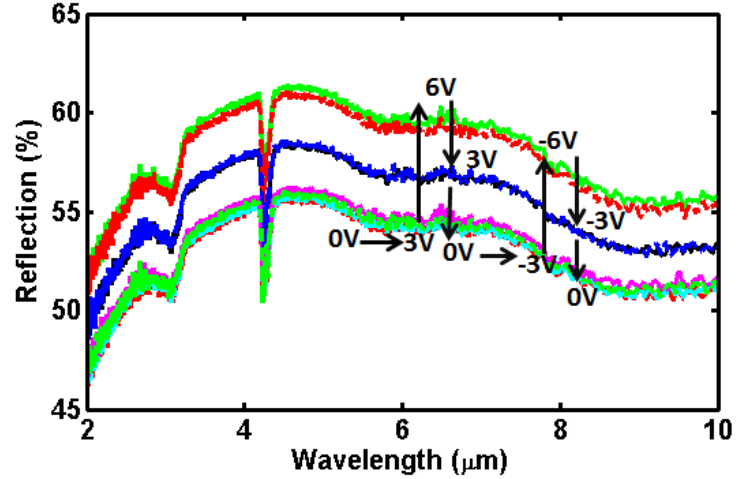

Figure 4 - Spectral response of the device under electrical bias. Time history of the bias voltage is shown with arrows starting from left to right. The upward and downward arrows indicate the change In the reflection spectra.

\section{ACKNOWLEDGEMENTS}

This work was supported in part by European Union Framework Program 7 Marie Curie IRG Grant 239444, COST NanoTP, TUBITAK Grants 109E044, 112M004 and 112E052. The authors acknowledge support from TUBITAK-BIDEB.

\section{REFERENCES}

[1] K. Diest, J. A. Dionne, M. Spain, and H. A. Atwater, Nano Letters, vol. 9, pp. 2579-2583, 2009/07/08 2009.

[2] E. Battal and A. K. Okyay, Opt. Lett., vol. 38, pp. 983-985, 2013.

[3] S. M. Menke, R. Pandey, and R. J. Holmes, Applied Physics Letters, vol. 101, pp. 223301-4, 2012.

[4] N. S. Holliman, N. A. Dodgson, G. E. Favalora, and L. Pockett, Broadcasting, IEEE Transactions on, vol. 57, pp. 362-371, 2011.

[5] A. E. Attard, Appl. Opt., vol. 38, pp. 3239-3248, 1999.

[6] J. F. Lotspeich, Spectrum, IEEE, vol. 5, pp. 45-52, 1968.

[7] A. Garzarella and W. Dong Ho, Ultra-Wideband (ICUWB), 2011

IEEE International Conference on, 2011, pp. 240-242.

[8] M. J. Dicken, K. Aydin, I. M. Pryce, L. A. Sweatlock, E. M. Boyd, S. Walavalkar, et al., Opt. Express, vol. 17, pp. 18330-18339, 2009.

[9] C. Qiu, X. Ye, R. Soref, L. Yang, and Q. Xu, Opt. Lett., vol. 37, pp. 3942-3944, 2012.

[10] A. Minovich, D. N. Neshev, D. A. Powell, I. V. Shadrivov, and Y. S. Kivshar, Applied Physics Letters, vol. 96, pp. 193103-3, 2010.

[11] L. Taelim, A. Higo, H. Fujita, Y. Nakano, and H. Toshiyoshi, Optical MEMS and Nanophotonics (OPT MEMS), 2010 International Conference on, 2010, pp. 107-108.

[12] E. Przeździecka, W. Ł, W. Paszkowicz, E. Łusakowska, T. Krajewski, G. Łuka, et al., Semiconductor Science and Technology, vol. 24, p. 105014, 2009. 